\title{
EVEN MULTIPLY PERFECT NUMBERS OF FIVE DIFFERENT PRIME FACTORS.
}

\author{
BY PROFESSOR R. D. CARMICHAEL.
}

(Read before the American Mathematical Society, April 27, 1907.)

THE problem of the existence of multiply perfect numbers has been completely solved for the cases in which the numbers contain not more than four different prime factors.* The object of this note is to give the results of a similar study of even multiply perfect numbers of five different prime factors. It will be found that there are three such numbers of multiplicity 4 and one of multiplicity 3 .

The lemma $\uparrow$ which formed the starting point of a previous paper will be repeated:

Lemma. If $x$ is a positive integer $>1, x^{t}-1$ has a prime factor not dividing $x^{u}-1(u<t)$, except in the cases $t=2$, $x=2^{v}-1, v \geqq 2 ; t=6, x=2$. Such prime factors of $x^{t}-1$ are of the form st +1 , and evidently if $t$ is odd and $>1$, they are of the form $2 s t+1$. factor.

Such a prime, $s t+1$ or $2 s t+1$, is called a characteristic

Throughout we shall represent the numbers by

$$
N=2^{a_{1}} p_{2}^{a_{2}} p_{3}^{\alpha_{3}} p_{4}^{a_{4}} p_{5}^{a_{5}},
$$

where $p_{2}<p_{3}<p_{4}<p_{5}$ are different odd primes. If further we denote the multiplicity by $m$, we have readily the following fundamental formulas:

$$
\begin{aligned}
& m=\frac{2^{a_{1}+1}-1}{2^{a_{1}}} \cdot \frac{p_{2}^{a_{2}+1}-1}{p_{2}^{a_{2}}\left(p_{2}-1\right)} \cdots \frac{p_{5}^{a_{5}+1}-1}{p_{5}^{a_{5}}\left(p_{5}-1\right)}, \\
& m<2 \cdot \frac{p_{2}}{p_{2}-1} \cdots \frac{p_{5}}{p_{5}-1} .
\end{aligned}
$$

From (3) it follows that $m \leqq 4$, as may be seen by substituting $3,5,7,11$ for the $p$ 's. But $m \neq 2$; for all even numbers

* Carmichael, Annals of Mathematics, ser. 2, vol. 8, pp. 49-56, 149-158.

† Carmichael, l. c., p. 151. 
of multiplicity 2 have only two prime factors.* Hence we have to consider the two cases $m=4, m=3$.

When $m=4$ it is easy by inequality (3) to show that $p_{2}=3$ and $p_{3}=5$ or 7 and also to restrict the values of $p_{4}$ and $p_{5}$. Then by the help of the lemma applied to expressions of the form $p^{a+1}-1$ the values of the exponents $a$ in (1) may be restricted. Then by an examination of the remaining possibilities I have found that equation (2) is never possible when $p_{3}=7$. On the other hand, when $p_{3}=5$, there are three cases when (2) is satisfied; and consequently three multiply perfect numbers of multiplicity 4 as follows: $2^{3} \cdot 3^{2} \cdot 5 \cdot 7 \cdot 13$, $2^{9} \cdot 3^{3} \cdot 5 \cdot 11 \cdot 31,2^{7} \cdot 3^{3} \cdot 5^{2} \cdot 17 \cdot 31$.

For $m=3$ a similar discussion restricts $p_{2}$ to have the value 3 or 5 . When $p_{2}=5$ equation (2) is impossible, as is shown again by examining the possibilities for $p_{3}, p_{4}, p_{5}$ and the exponents. When $p_{2}=3$ there is only one set of values satisfying $(2)$, and these give a perfect number $2^{13} \cdot 3 \cdot 11 \cdot 43 \cdot 127$, of multiplicity 3.

The results arrived at may be stated thus :

The even multiply perfect numbers of only five different prime factors are the following: $2^{3} \cdot 3^{2} \cdot 5 \cdot 7 \cdot 13,2^{9} \cdot 3^{3} \cdot 5 \cdot 11 \cdot 31$, $2^{7} \cdot 3^{3} \cdot 5^{2} \cdot 17 \cdot 31$ of multiplicity 4 ; and $2^{13} \cdot 3 \cdot 11 \cdot 43 \cdot 127$ of multiplicity 3.

All these numbers are included in the table published in the BulletiN, volume 13, pages 385-386. A misprint occurs in the table : 23569921 for 23569920.

alabama Presbyterian College,

ANNiston, Ala.

\section{THE FOURTH INTERNATIONAL CONGRESS OF MATHEMATICIANS: SECTIONAL MEETINGS.}

THE several sections of the Rome Congress had their first meetings on Tuesday morning, April 7. For each section one or more chairmen had been appointed to open the session, but at each the presiding officer was elected for the following day. In general those who opened the sessions merely welcomed the members and asked for nominations for president for that day. In each section a secretary was appointed who kept a

\footnotetext{
* Carmichael, l. c., p. 150.
} 